\title{
Veranstaltung des Berufsverbandes im Rahmen des virtuellen GTH-Kongresses 2021, aktuelle Corona-Sonderreglungen und Reimport von Gerinnungsfaktoren
}

\author{
Jürgen Koscielny ${ }^{1}$ Günther Kappert ${ }^{2}$ Christoph Sucker ${ }^{3,4}$ \\ ${ }^{1}$ Charité Universitätsmedizin Berlin, Germany \\ ${ }^{2}$ Gerinnungszentrum Rhein Ruhr, Duisburg, Germany \\ ${ }^{3}$ Medizinische Hochschule Brandenburg, Brandenburg an der Havel, \\ Germany \\ ${ }^{4}$ COAGUMED Gerinnungszentrum, Berlin, Germany
}

Hämostaseologie 2021;41:76-77.

\section{Einladung zur Veranstaltung des BDDH im Rahmen des virtuellen GTH-Kongresses 2021}

Der Vorstand des BDDH lädt alle Mitglieder zur Mitgliederversammlung des BDDH für Donnerstag, den 25.2.2021 von 15:00 bis 15:30 Uhr ein (nicht-öffentlicher Teil). Themen sind Bericht und Entlastung des Vorstandes sowie des Kassenwartes. Wahlen der Vorstandsmitglieder stehen im Rahmen der nächsten Mitgliederversammlung nicht an.

Davor findet am selben Tag von 14:00 bis 15:00 Uhr eine öffentliche Veranstaltung des BDDH statt, an der neben BDDH-Mitgliedern auch andere interessierte Kolleginnen und Kollegen teilnehmen können; Thema sind die „Auswirkungen der Gesetzesänderungen in Deutschland auf die Versorgung von Patienten mit hämophilen Gerinnungsstörungen in der täglichen Praxis“; den Vorsitz übernehmen Jürgen Koscielny als Vorsitzender und Günther Kappert als Stellvertretender Vorsitzender des BDDH. Die Situation wird aus der Sicht verschiedener Einrichtungen dargestellt, wobei folgende Beiträge geplant sind:

- Aus der Sicht eines Krankenhauses mit Maximalversorgung mit einem Hämophiliezentrum: R. Klamroth, Berlin

- Aus der Sicht einer Universitätsklinik mit einem Hämophiliezentrum: G. Goldmann, Bonn

- Aus der Sicht eines niedergelassenen MVZ mit einem Hämophiliezentrum: U. Scholz, Leipzig

- Aus der Sicht einer niedergelassenen Praxis mit einem Hämophiliekoordinationszentrum: W. Mondorf, Frankfurt/ Main

- Aus der Sicht eines niedergelassenen MVZ mit einem pädiatrischen Hämophiliezentrum: S. Halimeh, Duisburg

\section{Aktuelle Corona-Sonderregeln für die Leistungserbringung}

Für bestimmte ärztliche Leistungen gelten aufgrund der aktuellen Corona-Pandemie derzeit Sonderregeln, die den Praxisalltag in der epidemiologisch brisanten Situation zu vereinfachen.

So ist die Ausstellung oder Verlängerung von Arbeitsunfähigkeitsbescheinigungen/Krankmeldungen bei Erkrankungen der oberen Atemwege ohne schwere klinische Symptomatik auch nach telefonischem Kontakt von Patienten und Ärzten für eine Dauer von jeweils 7 Tagen möglich. Portokosten für die Zusendung von Bescheinigungen wie Arbeitsunfähigkeitsbescheinigungen und Folgerezepten sind abrechenbar. Auch die Verordnung von häuslicher Krankenpflege, Heil- und Hilfsmitteln sowie Krankenbeförderungen wurde vereinfacht.

\section{Informationen zum Reimportgeschäft für Gerinnungsfaktorenpräparationen}

Im Rahmen der bevorstehenden Verhandlungen zu Rabattverträgen auf Basis der Verträge zur ärztlichen Versorgung nach $\S 132$ i SGBV zwischen gesetzlichen Krankenkassen und den bekannten pharmazeutischen Unternehmen (PU) werden entsprechend den EU Gesetzen auch re-importierende Unternehmen teilnehmen. Diese werden entsprechend der EU-Gesetzgebung zahlreiche Gerinnungsfaktorenpräparationen pharmazeutischer Unternehmen aus anderen EULändern, in der Regel Reimporte, bereits ab dem 01.09.2020 auch an Apotheken und Großhandel vertreiben können.
(C) 2020. Thieme. All rights reserved. Georg Thieme Verlag KG,

Rüdigerstraße 14,

70469 Stuttgart, Germany
DOI https://doi.org/ 10.1055/a-1309-3253. ISSN 0720-9355. 
Reimporte sind Arzneimittel, die in Deutschland produziert und in andere EU-Länder exportiert wurden. Dort werden sie zu einem günstigeren Preis eingekauft und wieder nach Deutschland re-importiert. Weil sie im Ausland billiger sind als in Deutschland, kann der Re-Importeur sie zu einem Preis anbieten, der unter dem Produktpreis des direkt in Deutschland vertriebenen Präparates liegt. Diese Arzneimittel werden dadurch dem dortigen Markt des jeweiligen EU-Landes entzogen und dies kann unter Umständen dort zu Versorgungsengpässen führen.

Parallel importierte Präparate werden vom Hersteller in einem EU-Land produziert. So ist z. B. Irland aufgrund seiner niedrigen Unternehmenssteuersätze beliebt. Die Präparate werden dann in der Regel für die gesamte EU zugelassen und an die europäischen Tochtergesellschaften vertrieben.

Sind Präparate im Ausland günstiger als in Deutschland, kaufen Importeure diese im Ausland beim örtlichen PharmaGroßhandel ein und importieren sie nach Deutschland. Sowohl der Hersteller selbst als auch der Importeur importieren das Medikament also nach Deutschland. Dies ist die Regel, denn Produktionskosten und Unternehmenssteuern in anderen EU-Ländern sind oft niedriger als in Deutschland. Sehr viele „deutsche Original-Präparate“ sind daher tatsächlich auch Importe. In dieser Form gilt dies (noch) nicht für Gerinnungsfaktorenpräparationen.

Durch das (komplexe) Parallel- und Reimportgeschäft wird eine zusätzliche Tür geöffnet, die sich in einer Zunahme von Fälschungen in der regulären Lieferkette zeigt. Zum einen aufgrund der „gängigen“ Praxis des Umpackens zugelassener parallel-importierter Arzneimittel: Ein Arzneimittel, das zum Beispiel in Griechenland hergestellt wird, um in Deutschland (aufgrund der Preisdifferenz) teurer verkauft zu werden, muss umgepackt, eventuell zerschnitten, anders portioniert, umsortiert und mit deutschsprachigen Etiketten versehen werden. Hierdurch könnten die vom Originalhersteller aufgebrachten fälschungssicheren bzw. schützenden Maßnahmen zerstört werden. Zum anderen führt die zunehmende Anzahl von Groß- und Zwischenhändlern dazu, dass ein Arzneimittel, bevor es den Patienten erreicht, durch viele Hände an vielen Orten geht. Ein Vorbeileiten am eigentlichen Zielland und Verkauf in einem anderen Land, in dem höhere Preise locken, ist nicht auszuschließen. All diese Aspekte beeinflussen die Versorgungssysteme, was zu Engpässen führen und vor allem das Risiko für das Einschleusen von Fälschungen erhöhen kann.
Darum sollte nach wie vor jeder Hinweis von Patienten auf mögliche Qualitätsmängel auch bei diesen Waren sehr ernst genommen werden. Zudem ist zu vermeiden, dass mögliche zukünftige Look-Back-Verfahren nicht gefährdet werden oder eine Chargendokumentation nicht mehr eindeutig möglich ist.

In diesem Zusammenhang verweist der BDDH auf folgende geltende Regelungen in den Arzneiversorgungsverträgen, u.a. des VDEK und der AOK, die bei konsequenter Anwendung verhindern sollten, dass Gerinnungsfaktorenpräparationen seitens der Apotheken als Reimporte, z.B. Austausch auf Grund eines bestehenden Rabattvertrages, zum Patienten gelangen:

$\S 4$ Abs. 12 des Arzneiversorgungsvertrages der vdek-Kassen

Hat der Vertragsarzt ein Fertigarzneimittel unter seinem Produktnamen und/ oder seiner Pharmazentralnummer unter Verwendung des „aut-idem-Kreuzes“ verordnet, ist dies im Verhältnis von importiertem und Bezugsarzneimittel mangels arzneimittelrechtlicher Substitution unbeachtlich. Dies gilt nicht, wenn der Arzt vermerkt hat, dass aus medizinischtherapeutischen Gründen kein Austausch erfolgen darf.

$\S 4$ Abs. 11 des Arzneiversorgungsvertrages der AOK

Hat der Vertragsarzt ein Fertigarzneimittel unter seinem Produktnamen und/oder seiner Pharmazentralnummer unter Verwendung des „aut-idem-Kreuzes“ verordnet, ist dies im Verhältnis von importiertem und Bezugsarzneimittel mangels arzneimittelrechtlicher Substitution unbeachtlich. Dies gilt nicht, wenn der Vertragsarzt vermerkt hat, dass aus medizinisch-therapeutischen Gründen kein Austausch erfolgen darf.

Weitere Informationen diesbezüglich finden sich unter: https://www.apotheke-adhoc.de/nachrichten/detail/apo-5pp/import-und-original-fallen-und-regeln/

Aufgrund der o.g. Ausführungen empfiehlt der Vorstand des BDDH ALLEN seinen Mitgliedern zukünftig Rezepte für Gerinnungsfaktorenpräparationen mit dem Zusatz „aus medizinisch therapeutischen Gründen kein Austausch" zu versehen.

Für den Vorstand des Berufsverbandes der Deutschen Hämostaseologen e.V. (BDDH):

Priv.-Doz. Dr. Jürgen Koscielny, Berlin, Vorsitzender

Dr. Günther Kappert, Duisburg, Stellvertretender Vorsitzender

Priv.-Doz. Dr. Christoph Sucker, Berlin, Beisitzer des Vorstands 